Supplement of Geosci. Model Dev., 14, 3251-3268, 2021

https://doi.org/10.5194/gmd-14-3251-2021-supplement

(C) Author(s) 2021. CC BY 4.0 License.

(c) (i)

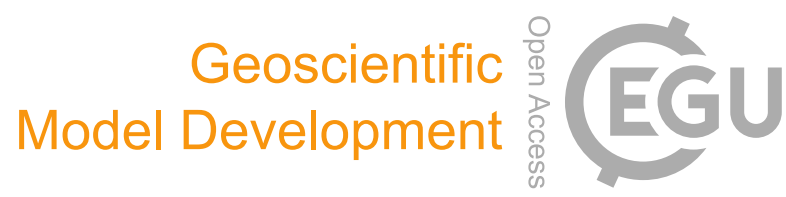

Supplement of

\title{
Simulation of $\mathrm{O}_{3}$ and $\mathrm{NO}_{x}$ in São Paulo street urban canyons with VEIN (v0.2.2) and MUNICH (v1.0)
}

\section{Mario Eduardo Gavidia-Calderón et al.}

Correspondence to: Mario Eduardo Gavidia-Calderón (mario.calderon@iag.usp.br)

The copyright of individual parts of the supplement might differ from the article licence. 


\section{Supplementary Material}

\section{Weighted emission factors}

We weighted emission factors, shown in Figure S1. It was calculated as a weighted mean with vehicles in circulation in 2011 and emission factors for 2011, both obtained from CETESB (2015).
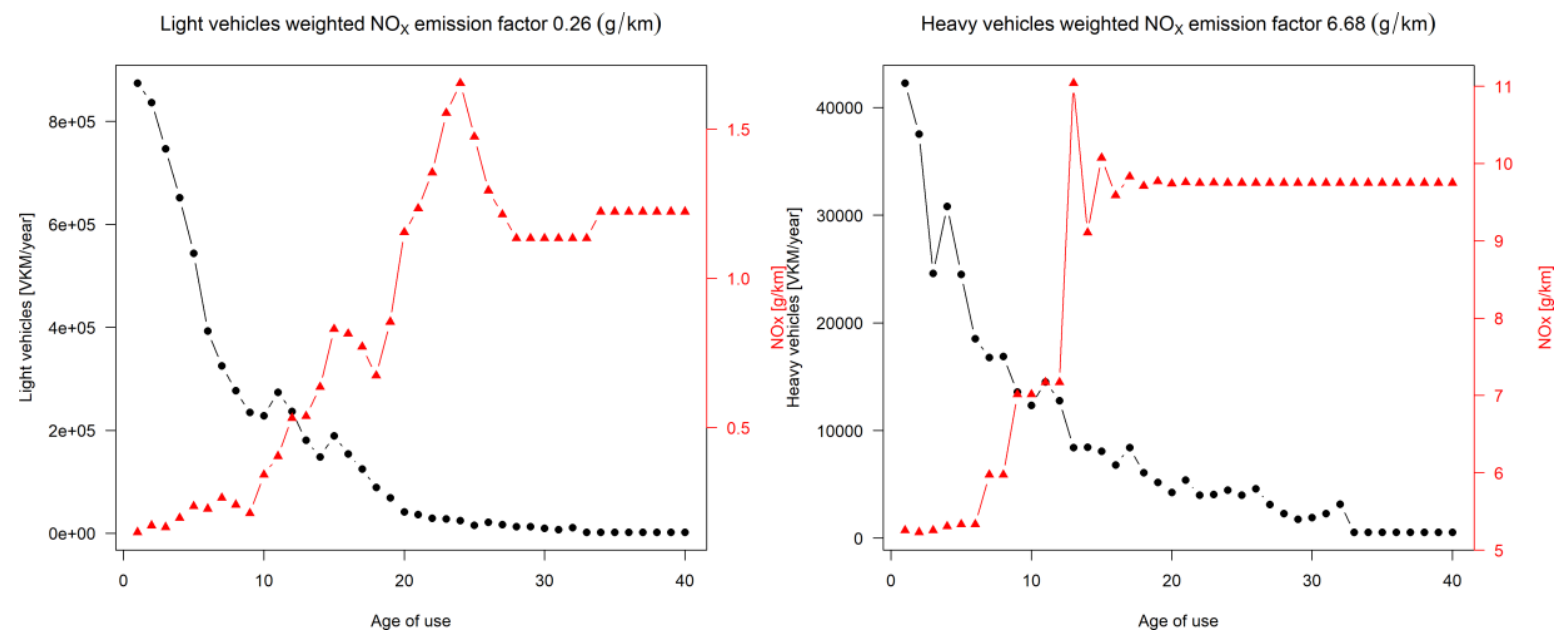

Figure S1. $\mathrm{NO}_{\mathrm{x}}$ weighted emission factors for light and heavy vehicles.

\section{VEIN typical week emissions for Pinheiros neighborhood}

Figure S2 shows the mean emission from all street links from the Pinheiros neighborhood for $\mathrm{NO}_{\mathrm{X}}$ and VOCs. For $\mathrm{NO}_{\mathrm{X}}$ emissions, Sunday total emissions are $25 \%$ lower than Saturday total emissions. For VOCs emissions, the values are almost the same between Saturday and Sunday. According to Ibarra et al. (2020), the difference between $\mathrm{NO}_{\mathrm{X}}$ emission during the weekday and the weekend is explained by the buses contribution, which is lower during the weekend, and even lower during Sunday. 
(a) NOX emissions at Pinheiros neghborhood

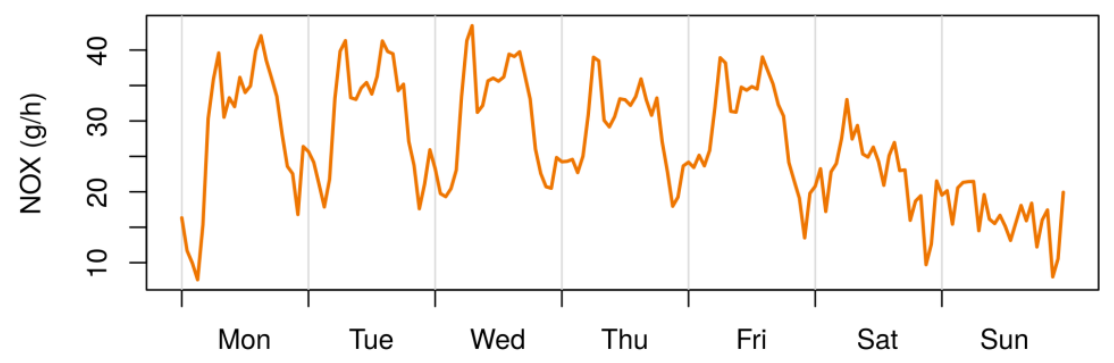

(b) VOCs emissions at Pinheiros neghborhood

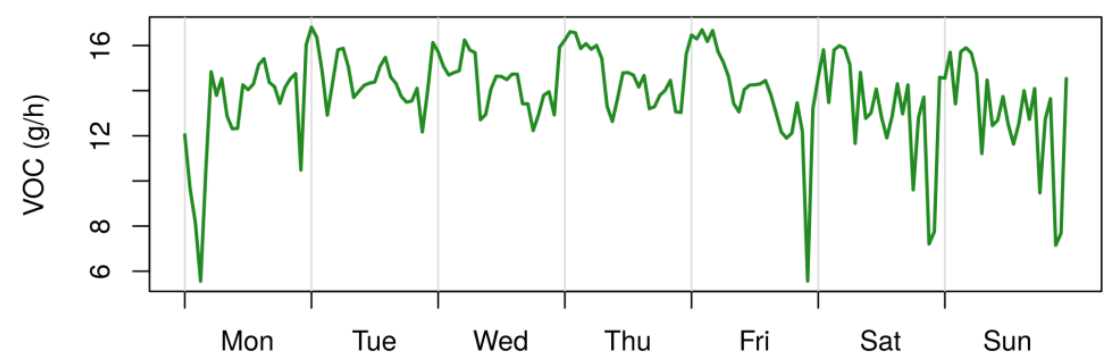

Figure S2. Mean emission from all street links from the Pinheiros neighborhood for (a) NOX and (b) VOCs for a typical week

\section{WRF simulation quality analysis}

To assess the quality of WRF simulation we calculate the statistical indicator in Table A1. The results are shown in Table S1. We then compare them with the recommended benchmark of Emery et al. (2001).

To calculate wind direction MB and MAGE we use the following equation based on Reboredo et al. (2015):

$$
\begin{gathered}
M B=\sum_{i=1}^{N} \frac{D}{N} \\
M A G E=\sum_{i=1}^{N} \frac{|D|}{N}
\end{gathered}
$$

If $\mathrm{Mi}<\mathrm{Oi}$ : 


$$
\begin{gathered}
D=\left(M_{i}-O_{i}\right) \text { if }\left|M_{i}-O_{i}\right|<\left|360+\left(M_{i}-O_{i}\right)\right| \\
D=360+\left(M_{i}-O_{i}\right) \text { if }\left|M_{i}-O_{i}\right|<\left|360+\left(M_{i}-O_{i}\right)\right|
\end{gathered}
$$

If $\mathrm{Mi}>\mathrm{Oi}$ :

$$
\begin{gathered}
D=\left(M_{i}-O_{i}\right) \text { if }\left|M_{i}-O_{i}\right|<\left|\left(M_{i}-O_{i}\right)-360\right| \\
D=\left(M_{i}-O_{i}\right)-360 \text { if }\left|M_{i}-O_{i}\right|<\left|\left(M_{i}-O_{i}\right)-360\right| \\
D=\left(M_{i}-O_{i}\right) \text { if }\left|M_{i}-O_{i}\right|<\left|\left(M_{i}-O_{i}\right)-360\right| \\
D=\left(M_{i}-O_{i}\right)-360 \text { if }\left|M_{i}-O_{i}\right|>\left|\left(M_{i}-O_{i}\right)-360\right|
\end{gathered}
$$

Further, according to Keyser and Anthes (1977) and Pielke (2013), model skill is estimated if It satisfies these criteria (Table S2):

1. $\sigma_{M} \cong \sigma_{O}$

2. $R M S E<\sigma_{O}$

3. $R M S E_{U B}<\sigma_{O}$

Where:

$$
\begin{gathered}
R M S E_{U B}=\sqrt{\sum_{i=1}^{N} \frac{\left[\left(M_{i}-\bar{M}\right)-\left(O_{i}-\bar{O}\right)\right]^{2}}{N}} \\
\sigma_{O}=\sum_{i=1}^{N} \frac{\left(O_{i}-\bar{O}\right)^{2}}{N} \\
\sigma_{M}=\sum_{i=1}^{N} \frac{\left(M_{i}-\bar{M}\right)^{2}}{N}
\end{gathered}
$$


Table S1. Statistical indicator for WRF simulation of T2, RH2, WS10 and WD10.

\begin{tabular}{lcccc}
\hline & $\mathrm{T} 2\left({ }^{\circ} \mathrm{C}\right)$ & $\mathrm{RH} 2(\%)$ & $\mathrm{WS} 10(\mathrm{~m} / \mathrm{s})$ & $\mathrm{WD} 10\left({ }^{\circ}\right)$ \\
\hline $\mathrm{N}$ & 1842 & 1843 & 1885 & 1864 \\
$\mathrm{FAC} 2$ & 1.00 & 0.99 & 0.67 & - \\
$\mathrm{MB}$ & 0.28 & -5.03 & 0.79 & -16.24 \\
MAGE & 1.60 & 9.73 & 1.17 & 55.08 \\
NMB & 0.01 & -0.08 & 0.43 & - \\
NMGE & 0.07 & 0.16 & 0.63 & - \\
RMSE & 1.98 & 12.79 & 1.52 & - \\
$\mathrm{R}$ & 0.94 & 0.85 & 0.45 & - \\
IOA & 0.83 & 0.74 & 0.18 & - \\
\hline
\end{tabular}

Table S2. Skill analysis for T2, RH2 and W10

\begin{tabular}{cccc}
\hline & $\mathrm{T} 2\left({ }^{\circ} \mathrm{C}\right)$ & $\mathrm{RH} 2(\%)$ & $\mathrm{WS} 10(\mathrm{~m} / \mathrm{s})$ \\
\hline $\bar{M}$ & 22.14 & 61.25 & 1.86 \\
$\sigma_{O}$ & 22.41 & 56.22 & 2.65 \\
$\sigma_{M}$ & 5.74 & 22.09 & 0.91 \\
$\mathrm{RMSE}$ & 4.98 & 19.71 & 1.41 \\
$\mathrm{RMSE}_{\mathrm{UB}}$ & 1.98 & 12.79 & 1.52 \\
$\sigma_{M} / \sigma_{O}$ & 1.96 & 11.76 & 1.40 \\
$R M S E_{U B} / R M S E$ & 0.87 & 0.89 & 1.55 \\
$R M S E_{U B} / \sigma_{O}$ & 0.99 & 0.92 & 0.92 \\
& 0.34 & 0.53 & 1.54 \\
\hline
\end{tabular}




\section{Wind field during daylight and nighttime}

We analyzed WRF model mean wind fields during daylight and nighttime to see if pollutants are advected from Ibirapuera (83) to Pinheiros (99) and Cerqueira Cesar (83) air quality stations. We can see that for our study period during daylight, when ozone is formed and present higher concentration, wind still present a South Easterly direction, which justifies the selection of Ibirapuera air quality station to provided background concentration to MUNICH. During nighttime, wind presents a westerly direction, but ozone concentrations are low.

(a)

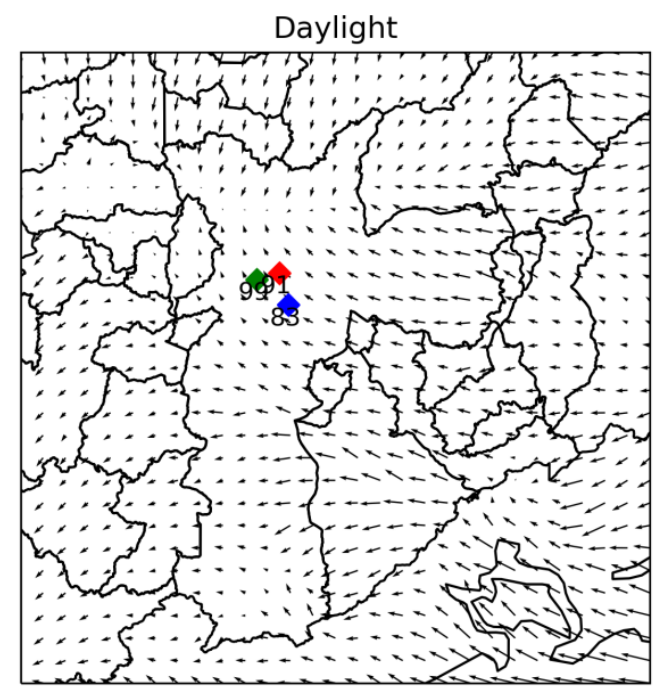

(b)

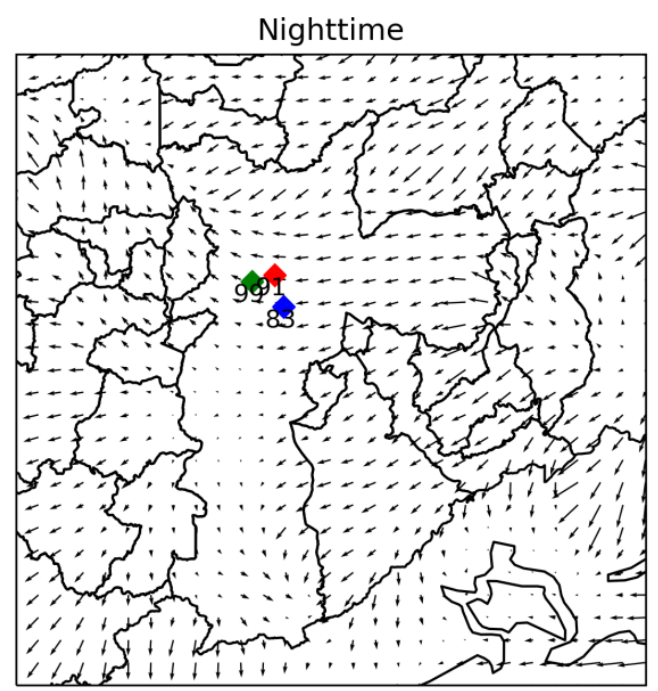

Figure S3. WRF averaged wind fields for daylight and nighttime during the simulation period. The green diamond shows Pinheiros AQS (99), the red diamond shows Cerqueira Cesar AQS (91), and the blue diamond shows Ibirapuera AQS (83)

\section{Test with another background concentration}

We perform a test by using measurements from a different AQS as MUNICH background information. We select Santos AQS (light blue diamond in Figure 4). This AQS recorded lower $\mathrm{O}_{3}$ concentration and higher NO concentrations than Ibirapuera AQS. Figure S4 shows a comparison of MUNICH results against background and observation concentrations for $\mathrm{O}_{3}, \mathrm{NO}_{\mathrm{x}}, \mathrm{NO}$, and $\mathrm{NO}_{2}$ and Figure $\mathrm{S} 5$ shows the diurnal profile. Table $\mathrm{S} 3$ shows the statistical indicator of the tests. 

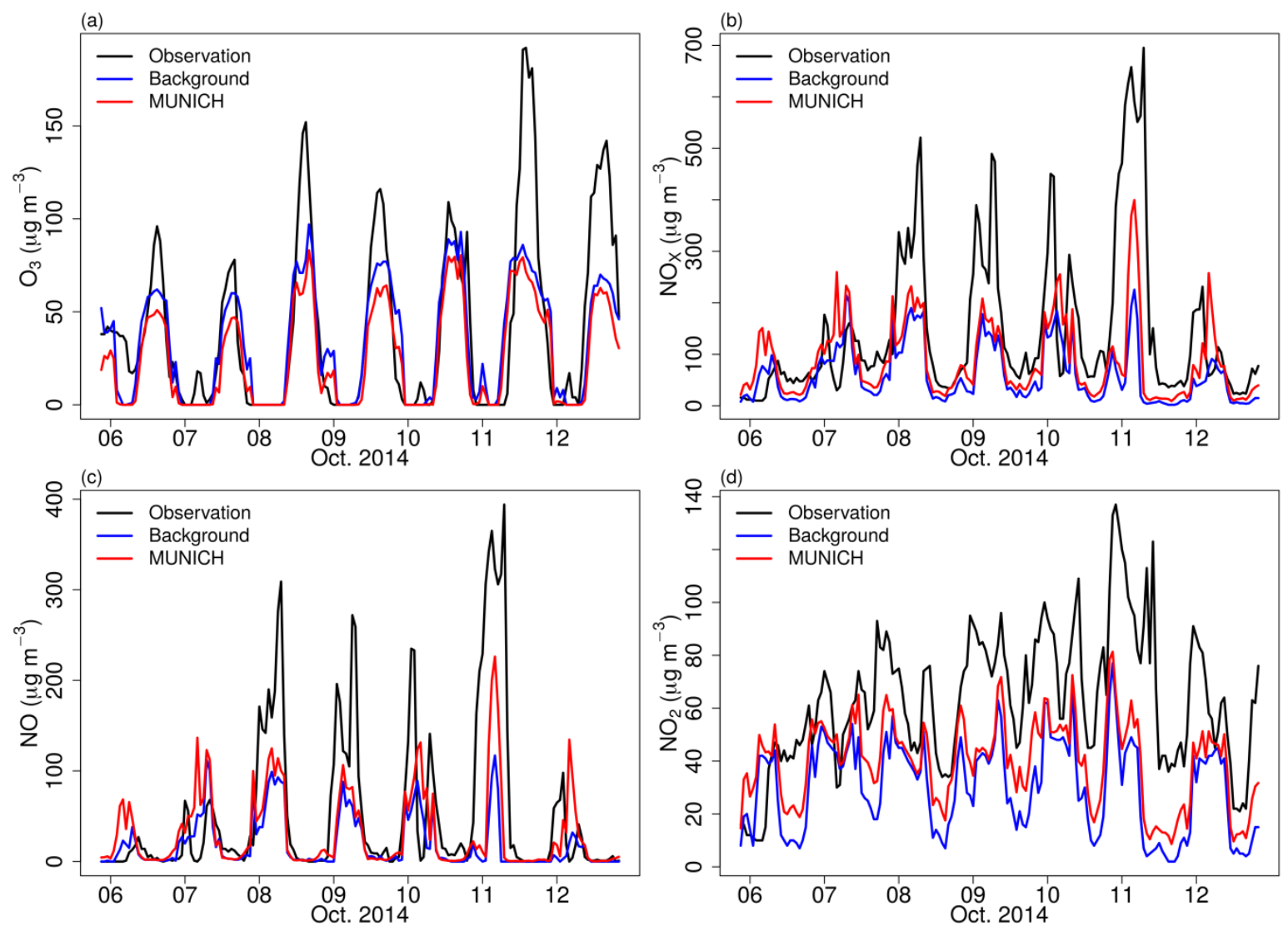

Figure S4. Comparison of MUNICH results against background and observation concentrations for (a) $\mathrm{O}_{3}$, (b) $\mathrm{NO}_{\mathrm{x}}$, (c) $\mathrm{NO}$, and (d) $\mathrm{NO}_{2}$ for Pinheiros urban canyon using Santos AQS measurements as background concentrations. 

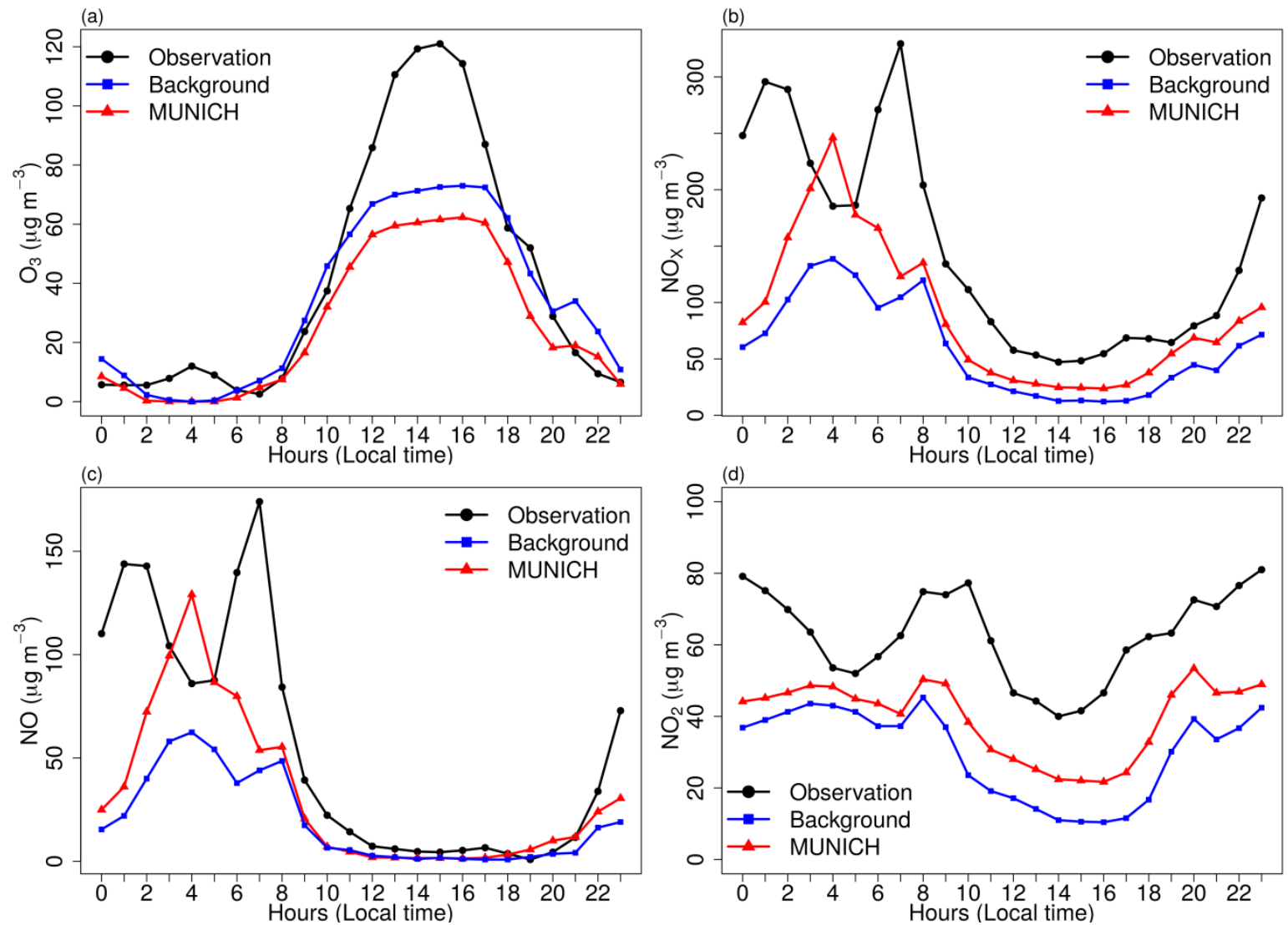

Figure S5. Diurnal profile of MUNICH results, background and concentration for (a) $\mathrm{O}_{3}$, (b) $\mathrm{NO}_{\mathrm{x}}$, (c) $\mathrm{NO}$, and (d) $\mathrm{NO}_{2}$ for Pinheiros urban canyon using Santos AQS measurements as background concentration.

Table S3. Statistical indicators for $\mathrm{O}_{3}, \mathrm{NO}_{\mathrm{x}}$, NO, and $\mathrm{NO}_{2}$ for comparison of MUNICH using Ibirapuera AQS as background and MUNICH using Santos AQS as background against observations from Pinheiros AQS.

\begin{tabular}{|c|c|c|c|c|c|c|c|c|c|c|c|c|c|c|}
\hline & & $\bar{M}^{\mathrm{a}}$ & $\bar{O}$ & $\sigma_{M}$ & $\sigma_{O}$ & MB & NMB & NMGE & RMSE & $\mathrm{R}$ & $|\mathrm{FB}|$ & NMSE & FAC2 & NAD \\
\hline \multirow[t]{2}{*}{$\mathrm{O}_{3}$} & MUNICH Ibi. & 54.5 & 41.5 & 62.1 & 47.5 & 13.0 & 0.3 & 0.3 & 22.2 & 1.0 & 0.3 & 0.2 & 0.6 & 0.1 \\
\hline & MUNICH San. & 25.7 & 41.5 & 26.9 & 47.5 & -15.8 & -0.4 & 0.5 & 32.5 & 0.8 & 0.5 & 1.0 & 0.4 & 0.2 \\
\hline \multirow[t]{2}{*}{$\mathrm{NO}_{\mathrm{X}}$} & MUNICH Ibi. & 88.9 & 146.4 & 57.4 & 150.3 & -57.4 & -0.4 & 0.5 & 128.5 & 0.7 & 0.5 & 1.3 & 0.7 & 0.2 \\
\hline & MUNICH San. & 88.4 & 146.4 & 75.4 & 150.3 & -57.9 & -0.4 & 0.6 & 137.3 & 0.6 & 0.5 & 1.5 & 0.5 & 0.2 \\
\hline \multirow[t]{2}{*}{ NO } & MUNICH Ibi. & 18.7 & 54.6 & 28.7 & 88.9 & -35.9 & -0.7 & 0.8 & 80.7 & 0.7 & 1.0 & 6.4 & 0.1 & 0.5 \\
\hline & MUNICH San. & 31.9 & 54.6 & 43.6 & 88.9 & -22.7 & -0.4 & 0.8 & 76.1 & 0.6 & 0.5 & 3.3 & 0.3 & 0.3 \\
\hline \multirow[t]{2}{*}{$\mathrm{NO}_{2}$} & MUNICH Ibi. & 45.8 & 62.7 & 23.4 & 25.9 & -16.8 & -0.3 & 0.3 & 21.2 & 0.9 & 0.3 & 0.2 & 0.9 & 0.2 \\
\hline & MUNICH San. & 39.5 & 62.7 & 15.9 & 25.9 & -23.1 & -0.37 & 0.4 & 32.1 & 0.5 & 0.5 & 0.4 & 0.7 & 0.2 \\
\hline
\end{tabular}

${ }^{\mathrm{a}} \bar{M}$ - Model value mean, $\bar{O}$ - Observation mean, $\sigma_{M}$ - model standard deviation, $\sigma_{O}$ - observation standard deviation, MB - mean bias, NMB - normalized mean bias, NMGE - normalized mean gross error, RMSE root mean square error, R - correlation coefficient, FB - fractional mean bias, NMSE - normalized meansquare error, FAC2 - fraction of predictions within a factor of two , and NAD - normalized absolute difference. Values in bold satisfied Hanna and Chang (2012) acceptance criteria. 


\section{$6 \mathrm{NO}_{\mathrm{x}}$ emission increase}

We conduct a sensitivity simulation in which $\mathrm{NO}_{\mathrm{x}}$ emissions are increased by four relative to the calibrated emission case, and maintaining VOCs emission as the original case scenario. Figure S6 shows a comparison of MUNICH results against background and observation concentrations for $\mathrm{O}_{3}, \mathrm{NO}_{\mathrm{x}}, \mathrm{NO}$, and $\mathrm{NO}_{2}$. Figure $\mathrm{S} 7$ shows the diurnal profile. Though there was an improvement in $\mathrm{O}_{3}$ simulation, improbable $\mathrm{NO}_{\mathrm{x}}$ concentrations are simulated, too. Table $\mathrm{S} 4$ shows the statistical performance indicator of this test.
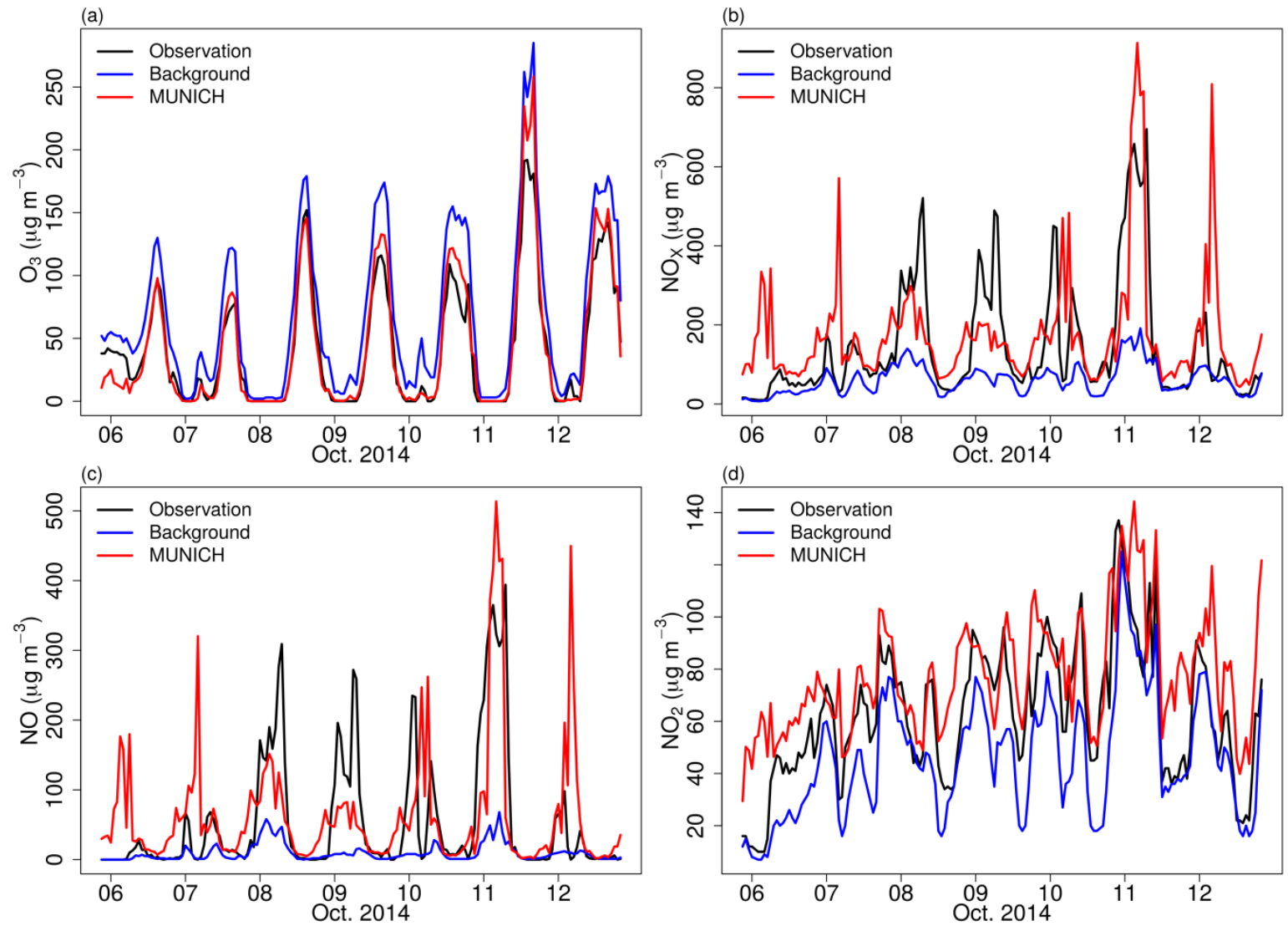

Figure S6. Comparison of MUNICH results against background and observation concentrations for (a) $\mathrm{O}_{3}$, (b) $\mathrm{NO}_{x}$, (c) NO, and (d) $\mathrm{NO}_{2}$ for Pinheiros urban canyon using increased $\mathrm{NO}_{x}$ emissions by four. 

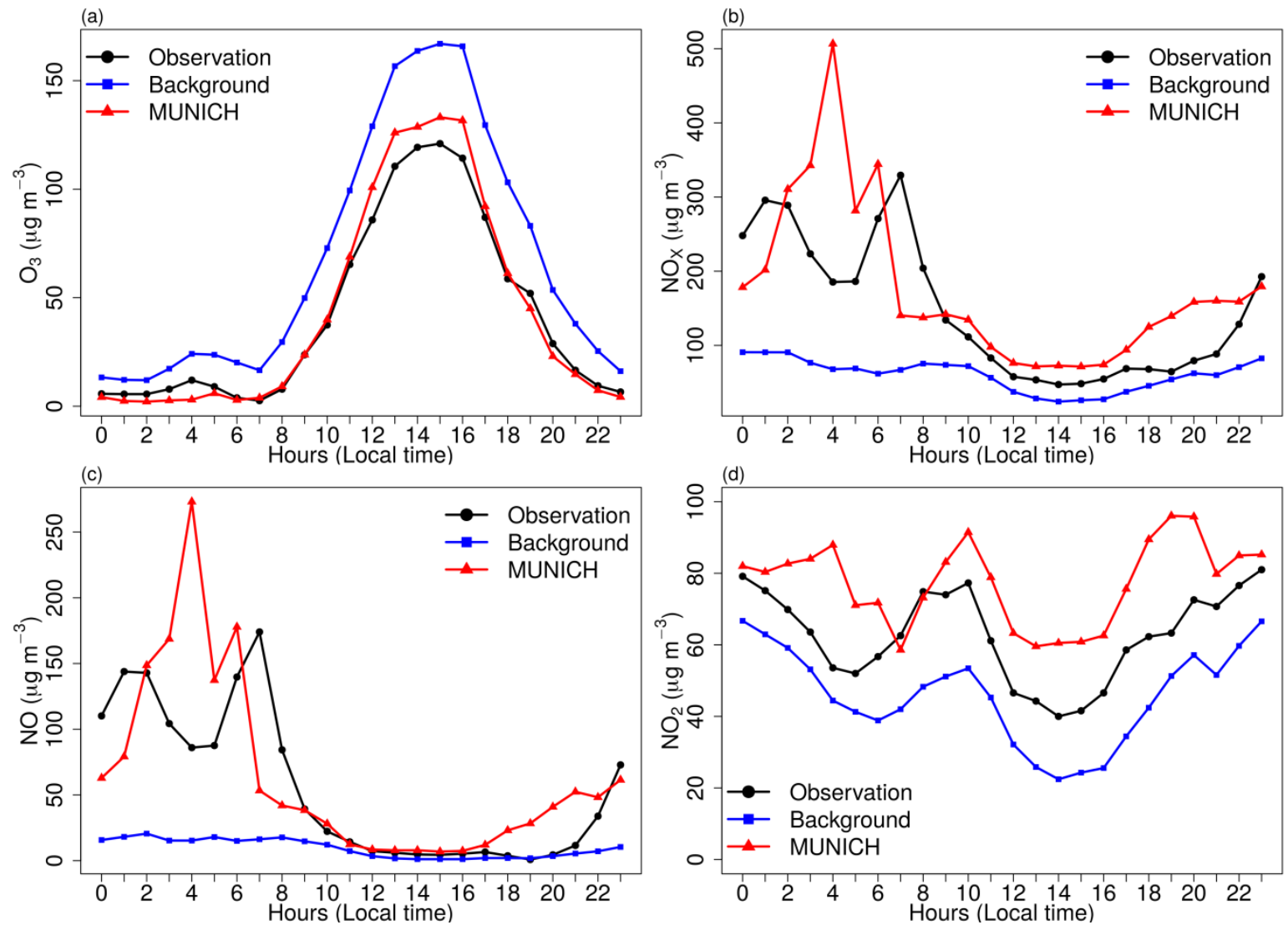

Figure S7. Diurnal profile of MUNICH results, background and concentration for (a) $\mathrm{O}_{3}$, (b) $\mathrm{NO}_{\mathrm{x}}$, (c) $\mathrm{NO}$, and (d) $\mathrm{NO}_{2}$ for Pinheiros urban canyon using increased $\mathrm{NO}_{\mathrm{x}}$ emissions by four.

Table S4. Statistical indicators for $\mathrm{O}_{3}, \mathrm{NO}_{\mathrm{x}}$, $\mathrm{NO}$, and $\mathrm{NO}_{2}$ for comparison of MUNICH using increased $\mathrm{NO}_{\mathrm{x}}$ emission by four.

\begin{tabular}{lrrrrrrrrrrrrr}
\hline & \multicolumn{1}{c}{$\bar{M}^{\mathbf{b}}$} & \multicolumn{1}{c}{$\bar{O}$} & \multicolumn{1}{c}{$\sigma_{M}$} & \multicolumn{1}{c}{$\sigma_{O}$} & \multicolumn{1}{c}{ MB } & NMB & NMGE & RMSE & R & FB & NMSE & FAC2 & NAD \\
\hline $\mathrm{O}_{3}$ & 43.2 & 41.5 & 54.8 & 47.5 & 1.7 & 0.0 & 0.2 & 13.6 & 1.0 & $\mathbf{0 . 0}$ & $\mathbf{0 . 1}$ & $\mathbf{0 . 6}$ & $\mathbf{0 . 0}$ \\
$\mathrm{NO}_{\mathrm{X}}$ & 175.0 & 146.4 & 147.4 & 150.3 & 28.6 & 0.2 & 0.6 & 146.5 & 0.5 & $\mathbf{0 . 2}$ & $\mathbf{0 . 8}$ & $\mathbf{0 . 6}$ & $\mathbf{0 . 1}$ \\
$\mathrm{NO}$ & 63.6 & 54.6 & 89.1 & 88.9 & 9.0 & 0.2 & 0.9 & 87.1 & 0.5 & $\mathbf{0 . 2}$ & $\mathbf{2 . 2}$ & $\mathbf{0 . 4}$ & $\mathbf{0 . 1}$ \\
$\mathrm{NO}_{2}$ & 77.4 & 62.7 & 22.2 & 25.9 & 14.8 & 0.2 & 0.3 & 22.7 & 0.8 & $\mathbf{0 . 2}$ & $\mathbf{0 . 1}$ & $\mathbf{0 . 9}$ & $\mathbf{0 . 1}$ \\
\hline
\end{tabular}

${ }^{\mathrm{b}} \bar{M}$ - Model value mean, $\bar{O}$ - Observation mean, $\sigma_{M}$ - model standard deviation, $\sigma_{O}$ - observation standard deviation, MB - mean bias, NMB - normalized mean bias, NMGE - normalized mean gross error, RMSE root mean square error, R - correlation coefficient, FB - fractional mean bias, NMSE - normalized meansquare error, FAC2 - fraction of predictions within a factor of two , and NAD - normalized absolute difference. Values in bold satisfied Hanna and Chang (2012) acceptance criteria. 